\title{
New Technique for Processing Tissue Displacements Estimation in Breast Ultrasound Elastography
}

Taher Slimi ${ }^{1 *}$, Ines Marzouk Moussa ${ }^{2,3}$, Tarek Kraiem ${ }^{3,4}$ and Halima Mahjoubi ${ }^{1}$

${ }^{1}$ Laboratory of Biophysics and Medical Technologies, High Institute of Medical Technologies of Tunis, University of Tunis El Manar, Tunis, Tunisia ${ }^{2}$ Department of Medical Imaging and Radiology, University Hospital Center of Monji Slim, Marsa, Tunisia

${ }^{3}$ Department of Biophysics, Faculty of Medicine of Tunis, University of Tunis El Manar, Rabta, Tunisia

${ }^{4}$ Department of National Radiation Protection Center, Bab Sadoun Children's Hospital, Tunisia

\begin{abstract}
Breast ultrasound elastography allows acquiring a displacements estimation images that reflects the tissue elasticity of the area under ultrasound examination. The displacements analysis of the mammary tissues helps to provide an accurate diagnosis related to tissue stiffness evaluation.

However; despite the fact that this imaging modality is promising and effective, it encounters a major problem, B-mode images are altered by speckle noise which comes from the difference in echogenicity of the imaged tissues, which deteriorates the diagnostic information, therefore, an application of displacements estimation methods on contaminated B-mode images, amplifies indirectly the speckle noise, causing a reduction of clinical evaluation capacity and prevention of rigorous clinical assessment.

In this perspective, the development of tools for treating this problem is crucial and indispensable for controlling the appearance of speckle noise. In this paper, we propose a new strategy based on the bilinear deformable block matching (BDBM) technique, in order to improve the old bilinear deformable block matching (OBDBM) technique by improving the B-mode images pre-processing step, at this stage, the B-mode images processing before estimating displacements is sufficient to have a good estimate. The proposed approach does not only filter the image against noise, it also improves the estimation of tissue deformations, the developed approach is based on the coupling between the reconstruction by filtered back projection (FBP) technique with shrinkage wavelets.
\end{abstract}

The proposed model was validated using in vivo database containing 20 B-mode images acquired before and after compression. The FBP approach was used to reconstruct the tissue texture, consequently improves the resolution and the contours erased by the noise, after that the shrinkage wavelets have been introduced to completely filter the image against speckle noise.

By comparing our proposed method with OBDBM and monogenic techniques (displacement estimation method based monogenic technique), we show that it is the most efficient in term of standard deviation calculation between the pixels (SD), it is better also for calculating the contrast to noise ratio (CNR) and it provides an excellent structural similarity (SSIM).

The results of the proposed technique are encouraging and it is ready to be used in mammary tissues displacements enhancement images for ultrasound elastography.

Keywords: Ultrasound elastography; Displacements estimation; Speckle noise

\section{Introduction}

Static ultrasound elastography is a promising medical imaging technique, used to analyze the tissue displacements. The use of this technique appears in medical imaging research in order to evaluate the rigidity of cellular lesions with different types, as the example of breast cancer; the analysis of mammary squirrhoeic breast carcinomas appear more rigid and less elastic than the surrounding soft mammary tissue [1]. In this context, the breast ultrasound elastography aim is to provide to clinicians the displacements of breast tissue in order to assess their rigidity; this technique supplies a quantification of tissue elastic criteria [2].

On the historical scale, doctors have used this technique qualitatively for a long time, by the principle of breast palpation. During palpation, doctor can evaluate the tissues stiffness, which helps to evaluate the tissues mechanical properties [3].

By this order of analogy between palpation and ultrasound technology, researchers concentrated their efforts to image the elastic tissues properties, by measuring tissue displacements estimation images
[4]. Clinically, imaging the breast surface with ultrasound probe, under compression and analyzing the induced deformations [5].

In the literature, researchers have developed several methods for estimating tissue displacements in ultrasound medical imaging; however, this technique presents a major problem that is related to noise and artifacts in the environment of the imaging system [6].

The use of any technique to estimate tissues displacements suffers

*Corresponding author: Taher Slimi, Laboratory of Biophysics and Medica Technologies, High Institute of Medical Technologies of Tunis, University of Tunis El Manar, Zouhair Essafi Street, Tunis, Tunisia, Tel: 0021622352 994; E-mail: slimi.taher@hotmail.com

Received December 04, 2017; Accepted December 31, 2017; Published January 06, 2018

Citation: Slimi T, Moussa IM, Kraiem T, Mahjoubi H (2018) New Technique for Processing Tissue Displacements Estimation in Breast Ultrasound Elastography. J Tissue Sci Eng 9: 214. doi: 10.4172/2157-7552.1000214

Copyright: ( 2018 Slimi T, et al. This is an open-access article distributed under the terms of the Creative Commons Attribution License, which permits unrestricted use, distribution, and reproduction in any medium, provided the original author and source are credited. 
from the presence of noise and artefacts deteriorating the tissues details and texture which leading to loss the image contrast, thus decreasing clinical evaluation capacity.

Therefore, the different methods of tissues displacements estimation images should perform appropriate tools to ultrasound images in a general manner and to ultrasound elastography images in a specific manner [7].

In this paper, we proposed a new technique that improves the OBDBM method. We have shown how the proposed approach improves the estimation of mammary tissues displacements. The proposed new approach is based on the FBP coupled to the shrinkage wavelets combined with the BDBM model. This proposed strategy optimizes the OBDBM method used in the displacement of the breast tissues.

We have also validated our method on 20 B-mode images corresponding to 20 patients with mammary tumors. We showed that the proposed displacements estimation method is more accurate than both OBDBM and monogenic methods.

The performance of our proposed approach is also measured and compared to the above cited method; we showed that our approach is more efficient, we show that it is the most efficient in term of SD, it is better also for calculating the CNR and it provides an excellent SSIM.

The following section presents our adapted filtering technique and the BDBM theory of displacement estimation.

The third section shows the results of improvement of the images of tissue displacements of the breast by the proposed model, we also evaluated the quality of the images obtained qualitatively and quantitatively, comparing them to the OBDBM and monogenic techniques. In the fourth part, we analyzed and discussed the obtained results.

The last part is left to the conclusion remarks.

\section{Methods}

\section{Displacement estimation enhancement based on BDBM method}

Image filtering: Speckle noise is a common phenomenon in ultrasonic imaging, its origin is attributed to the random interference, between the coherent return, from the different diffusers, present in the structure tissue of the breast [8].

Speckle noise is an undesirable effect, considering its characteristics to alter the quality of the elastographic image. Therefore, speckle reduction proves to be a pre-treatment step for a good clinical evaluation [9].

The main idea to restore the lost details in image, like loss of resolution and loss of clinical diagnosis information; is to reconstruct the breast B-mode images, in order to improve sharpness and restore tissue structure, after that, we implement a filtering step to reduce noise and artefacts [10].

Filtered back projection: In the literature, researchers have developed several techniques for lost information processing in ultrasound elastography, among these powerful algorithms: we propose the FBP technique, which has proved its effectiveness in the correct tracing of the details that are altered by speckle noise and artefacts [11]. The B-mode images are covered by a multiplicative distribution of speckle noise which has greatly decreased the performance of ultrasonic elastography. In this context, the FBP has potentially solved this problem by projection and texture filtered back projection process of B-mode images, whose purpose is to improve the sharpness and the visibility of effaced contours.

Following the theory of Radon who claimed that to restore the invisible details of an object we reconstruct it by a projection technique, and then we apply the back projection. This theory is modelled mathematically by the following expression [12]:

$$
\mathrm{R}[\mathrm{f}(\mathrm{x}, \mathrm{y})]=\mathrm{p}(\mathrm{u}, \theta)=\int_{0}^{\pi} \mathrm{f}(\mathrm{x}, \mathrm{y}) d v
$$

The B-mode image is defined by $\mathrm{f}(\mathrm{x}, \mathrm{y})$, placed in the plane $(\mathrm{x}, \mathrm{y})$ The projections made are defined by "p". The axis " $v$ " is perpendicular to the axis " $u$ " constituting the base of summation of the projections on the $\mathrm{B}$-mode image. The projection angle is defined by $\theta$.

We note that during the FBP step, there is a creation of the artefacts, which depends on the projection numbers, causing a loss of the image contrast. The cause of occurrence of these added artefacts during projection step is related to the presence of the residuals projections in areas where no signal exists in image, this slight problem is managed by filtering the image in the Fourier transform domain. In this axis, we propose the use of the Ramp filter followed by the Hamming filter, in order to reduce the induced artefacts [13].

The filtering corresponding to the Ramp filter is defined by the following equation

$$
p^{\prime}(\mathrm{u}, \theta)=\int_{-\infty}^{+\infty} P(q, \theta)|q| \cdot e^{2 i \pi q u} d q
$$

Where $\mathrm{p}$ is a filtered sinogram (image obtained after projection) and " $\mathrm{q}$ " is the filter response.

The Hamming filter is implemented then to be combined with the Ramp filter, the filter window is defined by the following expression:

$$
W(q)\left\{\begin{array}{c}
=0.54+0.46\left(\cos \pi f / f_{c}\right) \text { sif }<f_{c} \\
=0 \text { sif } \geq f_{c}
\end{array}\right.
$$

Where $\mathrm{fc}_{\mathrm{c}}$ is the cut-off frequency and $\mathrm{f}$ is the texture frequency.

After filtering, the image is reconstructed by back-projection process; the associated mathematical expression is defined by:

$$
F(x, y)=\int_{0}^{\pi}(u, \theta) d \theta
$$

Where $\mathrm{F}(\mathrm{x}, \mathrm{y})$ constitutes the filtered back projected B-mode image.

Shrinkage wavelets: After reconstructing the B-mode images and improving their visualization, it is ready to be filtered from the speckle noise. Several studies have been carried out to develop the efficiency of shrinkage wavelets in the treatment of speckle noise for ultrasound images, in this paper we adopt the use of this approach for the B-mode images denoising, using a Bayesian maximum a posteriori estimate, for more details concerning the use of this technique, we have detailed the use of this technique suitable for ultrasound imaging [14].

$$
\hat{g}=\left\{\begin{array}{l}
0 f \leq T_{k} \\
\operatorname{sign}(f) \cdot \max \left(|f|-\frac{\sigma_{n}^{2}+\sqrt{\sigma_{n}^{4}+2 \sigma_{n}^{2} \sigma_{g}^{2}}}{\sqrt{2 \sigma_{g}}}\right)
\end{array}\right.
$$

Where $\mathrm{g}$ is the image to be estimated, $\mathrm{f}$ is suspected in phase with noise-free signal g. sign (f) Designate symbolic function. 
Citation: Slimi T, Moussa IM, Kraiem T, Mahjoubi H (2018) New Technique for Processing Tissue Displacements Estimation in Breast Ultrasound Elastography. J Tissue Sci Eng 9: 214. doi: 10.4172/2157-7552.1000214

Page 3 of 5

$\mathrm{T}$ is a threshold, $\mathrm{k}(1,2, \ldots, \mathrm{K})$ are the decomposition layers of wavelet transformation, $\mathrm{K}$ denotes the largest decomposition layers, $\sigma \mathrm{n}$ denotes the standard deviation of noise in wavelet domain.

\section{Image filtering BDBM model}

After having filtered the two B-mode images, we integrated them into a BDBM model for displacements estimation algorithm. This technique estimates the displacements between the two acquired B-mode images (Before and after tissues deformation); the two motion components denoted $\mathrm{u}(\mathrm{x} 1, \mathrm{x} 2)$ and $\mathrm{v}(\mathrm{x} 1, \mathrm{x} 2)$, represent the dense motion field, as shown in the following expression $[15,16]$.

$$
\mathrm{i}_{2}\left(\mathrm{x}_{1}, \mathrm{x}_{2}\right)=\mathrm{i}_{1}\left(\mathrm{x}_{1}+\mathrm{u}\left(\mathrm{x}_{1}, \mathrm{x}_{2}\right), \mathrm{x}_{2}+\mathrm{v}\left(\mathrm{x}_{1}, \mathrm{x}_{2}\right)\right)
$$

In each pixel to be estimated in the affected bilinear model, a search block is selected in the target image. A simulation check criterion is used to find the best block candidate in the search block. The search block was defined by this expression with region of interest ROI size: $\mathrm{L}_{1} \times \mathrm{L}_{2}$.

Then we determine the position of the estimated block by calculating the maximum value of the inter-correlation coefficient (CC) [17].

$$
C C(p, d)=\sum_{x_{1}=-\frac{L_{1}}{2}}^{\frac{L_{1}}{2}} \sum_{x_{2}=-\frac{L_{2}}{2}}^{\frac{L_{2}}{2}} \mathrm{i}_{1}\left(P_{1}+x_{1} P_{2}+x_{2}\right) \mathrm{i}_{2}\left(P_{1}+x_{1}, d_{1} P_{2}+x_{2}+d_{2}\right)
$$

Centered on the pixel coordinates $\mathrm{P}=(\mathrm{P} 1, \mathrm{P} 2)$ in the original image. The deformation between a condidat pixel and the ROI of the original image is denoted $\mathrm{d}=(\mathrm{d} 1, \mathrm{~d} 2)$ and is limited by the size of the search block. The position of the best candidate block is denoted $\left(\hat{d}_{1}, \hat{d}_{2}\right)$ [18].

\section{Results}

In this section, all results have been verified and validated by three radiologist doctors.
The proposed method was implemented using Matlab software (The MathWorks, Matlab software, Pentium 4, 3.2 GHz); the radiologist set the frequency of $15 \mathrm{MHz}$. The proposed method for improving the displacements estimation was tested on a database containing 20 breasts B-mode images.

\section{In vivo breast images}

The improvement strategy described in Section 2 is evaluated here; we use the in vivo mammary B-mode images of 20 patients with breast tumor, acquired with clinical ultrasound scanner (Logiq E9), with 15 $\mathrm{MHz}$ linear probe (GE Healthcare).

The breast B-mode images were taken by the radiologist applying a small compression. We presented below the results of our proposed method for the displacements estimation enhancement compared to OBDBM and monogenic techniques (Figure 1) for one patient.

In order to confirm the occurrence and the effectiveness of our proposed technique, we measured, analyzed and evaluated its performance to OBDBM and monogenic techniques using quantitative criteria such as SD (Table 1), CNR comparison (Table 2) and SSIM index (Table 3).

\section{Discussion}

According to the experimental results presented in sections 3 , we can make a comparison between the previously mentioned methods and analyze the breast tissue displacements images, in this perspective we compared the results obtained by our proposed method with those obtained By the OBDBM and monogenic techniques. Comparison of results is based on the evaluation of qualitative and quantitative criteria for 20 tissue displacements images with mammary tumors. We note that all results have been verified and validated by three radiologists.

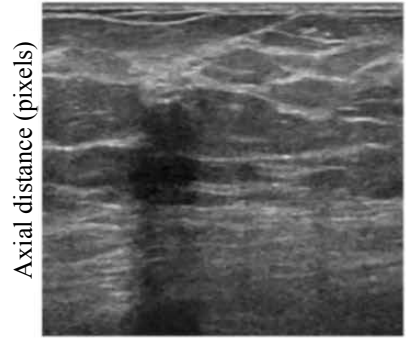

Lateral distance (pixels)

(a)

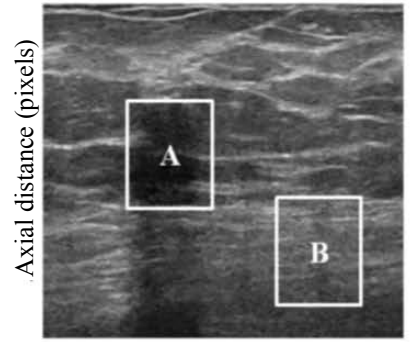

Lateral distance (pixels)

(b)

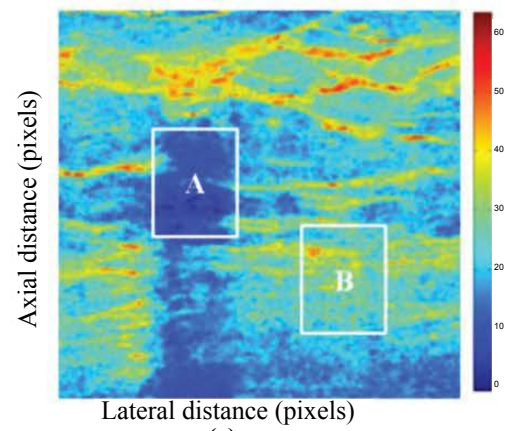

(c)

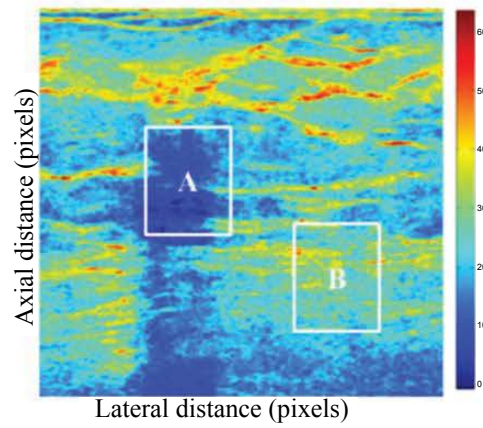

(d)

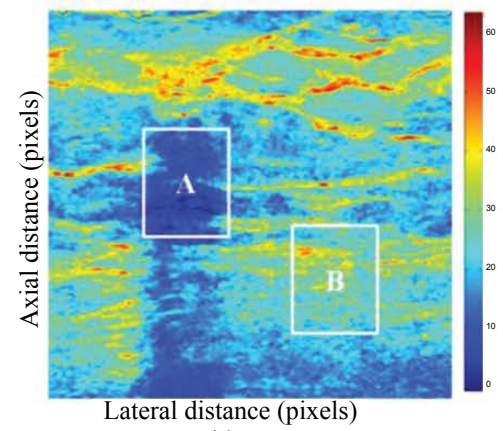

(e)

Figure 1: Estimating tissue displacements of breast tumor. A) Simulated pre compression B-mode image. b) Simulated post compression B-mode image. c) Tissues displacement obtained with OBDBM method. d) Tissues displacement obtained with monogenic method, and e) Tissues displacement obtained with proposed method: Areas selected by a rectangle are used for mathematical computation. 
Citation: Slimi T, Moussa IM, Kraiem T, Mahjoubi H (2018) New Technique for Processing Tissue Displacements Estimation in Breast Ultrasound Elastography. J Tissue Sci Eng 9: 214. doi: 10.4172/2157-7552.1000214

Page 4 of 5

\begin{tabular}{|l|c|c|c|}
\hline & $\begin{array}{c}\text { OBDBM } \\
\text { method }\end{array}$ & $\begin{array}{c}\text { Monogenic } \\
\text { method }\end{array}$ & $\begin{array}{c}\text { Proposed } \\
\text { method }\end{array}$ \\
\hline SD in pixels: Patient 1 & 4.91 & 3.87 & 3.01 \\
\hline SD in pixels: Patient 2 & 5.12 & 4.90 & 4.13 \\
\hline SD in pixels: Patient 3 & 6.11 & 5.30 & 4.99 \\
\hline SD in pixels: Patient 4 & 4.20 & 3.92 & 3.10 \\
\hline SD in pixels: Patient 5 & 3.98 & 2.93 & 1.98 \\
\hline SD in pixels: Patient 6 & 7.15 & 6.17 & 5.51 \\
\hline SD in pixels: Patient 7 & 3.41 & 5.18 & 4.87 \\
\hline SD in pixels: Patient 8 & 4.18 & 3.21 & 2.97 \\
\hline SD in pixels: Patient 9 & 2.31 & 1.20 & 1.11 \\
\hline SD in pixels: Patient 10 & 4.15 & 3.77 & 3.21 \\
\hline SD in pixels: Patient 11 & 6.70 & 5.98 & 4.90 \\
\hline SD in pixels: Patient 12 & 3.80 & 2.76 & 2.27 \\
\hline SD in pixels: Patient 13 & 7.18 & 6.71 & 5.93 \\
\hline SD in pixels: Patient 14 & 5.67 & 4.13 & 3.69 \\
\hline SD in pixels: Patient 15 & 4.52 & 4.98 & 3.81 \\
\hline SD in pixels: Patient 16 & 5.64 & 4.97 & 3.71 \\
\hline SD in pixels: Patient 17 & 4.81 & 3.21 & 2.96 \\
\hline SD in pixels: Patient 18 & 3.95 & 3.14 & 2.83 \\
\hline SD in pixels: Patient 19 & 7.91 & 6.32 & 5.96 \\
\hline SD in pixels: Patient 20 & 8.55 & 7.33 & 6.75 \\
\hline Tah 1. & & & \\
\hline
\end{tabular}

Table 1: In vivo results comparison of SD in pixels for the proposed method with OBDBM and monogenic methods for 20 patients.

\begin{tabular}{|l|c|c|c|c|}
\hline & $\begin{array}{c}\text { B-mode } \\
\text { image }\end{array}$ & $\begin{array}{c}\text { OBDBM } \\
\text { method }\end{array}$ & $\begin{array}{c}\text { Monogenic } \\
\text { method }\end{array}$ & $\begin{array}{c}\text { Proposed } \\
\text { method }\end{array}$ \\
\hline CNR: Patient 1 & 0.62 & 1.10 & 1.16 & 1.23 \\
\hline CNR: Patient 2 & 0.18 & 0.23 & 0.27 & 0.31 \\
\hline CNR: Patient 3 & 0.66 & 0.71 & 0.82 & 0.91 \\
\hline CNR: Patient 4 & 1.83 & 1.91 & 1.98 & 2.02 \\
\hline CNR: Patient 5 & 5.18 & 5.61 & 5.71 & 5.88 \\
\hline CNR: Patient 6 & 0.74 & 0.87 & 0.91 & 1.07 \\
\hline CNR: Patient 7 & 0.58 & 0.62 & 0.72 & 0.97 \\
\hline CNR: Patient 8 & 0.34 & 0.45 & 0.56 & 0.68 \\
\hline CNR: Patient 9 & 0.64 & 0.73 & 0.84 & 0.96 \\
\hline CNR: Patient 10 & 0.78 & 0.81 & 0.93 & 1.16 \\
\hline CNR: Patient 11 & 0.91 & 1.10 & 1.18 & 1.34 \\
\hline CNR: Patient 12 & 0.73 & 0.83 & 0.91 & 1.12 \\
\hline CNR: Patient 13 & 1.17 & 1.21 & 1.26 & 1.31 \\
\hline CNR: Patient 14 & 0.96 & 1.11 & 1.19 & 1.23 \\
\hline CNR: Patient 15 & 1.14 & 1.21 & 1.31 & 1.43 \\
\hline CNR: Patient 16 & 0.65 & 0.74 & 0.87 & 0.93 \\
\hline CNR: Patient 17 & 0.19 & 0.23 & 0.42 & 0.52 \\
\hline CNR: Patient 18 & 0.23 & 0.45 & 0.71 & 0.85 \\
\hline CNR: Patient 19 & 0.22 & 0.31 & 0.45 & 0.64 \\
\hline CNR: Patient 20 & 0.17 & 0.21 & 0.28 & 0.31 \\
\hline Table 2: Comparis & & & & \\
\hline
\end{tabular}

Table 2: Comparison of CNR for the proposed method with OBDBM and monogenic methods for 20 patients.

The analysis of Figure 1 shows that displacements estimation images in the case of our proposed approach seems very clear with a strong reduction of speckle noise, tissue contours well traced and well cared for good preservation of breast tissue texture. The proposed model also shows the good remarkable localization of the tumor, the inclusions are clearly visible, less artefacts and less noises compared to the results obtained by the OBDBM and monogenic techniques.

For a quantitative results evaluation, the SD, CNR and SSIM are calculated for each method.

\begin{tabular}{|l|c|c|c|}
\hline & OBDBM method & $\begin{array}{c}\text { Monogenic } \\
\text { method }\end{array}$ & $\begin{array}{c}\text { Proposed } \\
\text { method }\end{array}$ \\
\hline SSIM: Patient 1 & 0.90 & 0.94 & 1.09 \\
\hline SSIM: Patient 2 & 0.45 & 0.61 & 0.72 \\
\hline SSIM: Patient 3 & 0.76 & 0.79 & 0.83 \\
\hline SSIM: Patient 4 & 0.91 & 1.12 & 1.21 \\
\hline SSIM: Patient 5 & 0.16 & 0.31 & 0.52 \\
\hline SSIM: Patient 6 & 0.75 & 0.83 & 0.93 \\
\hline SSIM: Patient 7 & 0.74 & 0.91 & 1.19 \\
\hline SSIM: Patient 8 & 0.83 & 0.93 & 1.21 \\
\hline SSIM: Patient 9 & 1.51 & 1.61 & 1.78 \\
\hline SSIM: Patient 10 & 0.64 & 0.75 & 0.86 \\
\hline SSIM: Patient 11 & 0.63 & 0.73 & 0.97 \\
\hline SSIM: Patient 12 & 1.12 & 1.17 & 1.31 \\
\hline SSIM: Patient 13 & 1.76 & 1.80 & 1.94 \\
\hline SSIM: Patient 14 & 0.84 & 0.95 & 0.98 \\
\hline SSIM: Patient 15 & 1.74 & 1.85 & 1.95 \\
\hline SSIM: Patient 16 & 0.53 & 0.74 & 0.83 \\
\hline SSIM: Patient 17 & 0.69 & 0.79 & 0.81 \\
\hline SSIM: Patient 18 & 1.16 & 1.21 & 1.33 \\
\hline SSIM: Patient 19 & 0.79 & 0.86 & 0.91 \\
\hline SSIM: Patient 20 & 0.42 & 0.53 & 0.59 \\
\hline Table 3: Compar & & & \\
\hline
\end{tabular}

Table 3: Comparison of SSIM for the proposed method with OBDBM and monogenic methods for 20 patients.

It can be seen from Table 1, that the SD between the pixels of the proposed method is the best compared to OBDBM and monogenic techniques. Through the use of reconstruction and filtering technique, the breast texture was reconstructed without loss of diagnostic information with more precision. In addition, by integrating the shrinkage wavelets, we opted to reduce speckle noise in the image with good tissue preservation. This explains the smallest values found by the proposed model.

Unlike the conventional OBDBM technique, which has no B-mode images pre-processing step, the non-availability of filtering processes in the OBDBM technique favors an induced speckle noise amplification which deteriorates the images quality and decreases clinical evaluation capacity.

Thus the monogenic technique marks an unsatisfactory SD value compared to the proposed technique, this is explained by the insufficient capacity of the difference of Gaussian (DoG) filter implemented in this technique $[19,20]$, the implemented filtering did not succeed in totally eliminating the noise of the speckle, influenced the nature of the estimate. Therefore the proposed method is the best one used for a good estimate.

Thanks to the use of FBP and shrinkage wavelets algorithm which eliminates artefacts, parasites and enhances the sharpness of the image, coupled with the BDBM model which estimates the deformation with preserving the information necessary for medical diagnosis.

Nor the OBDBM method that it has no filtering process, can estimate accurately the sub pixel movement, neither can the monogenic technique method provides accurate displacements estimation, ahead of the proposed platform.

Another fundamental criterion has been studied: the CNR which represents a criterion very involved in the evaluation of ultrasound elastography modalities.

From Table 2, the tissue displacements estimation obtained by the 
proposed method shows a higher level of CNR than those obtained with other methods, with perfect details preservation. This superiority is explained by the fact that the CNR is improved by two steps in the developed model; the first step is related to the presence of the FBP which improve the contrast, by tracing the structures and reinforcing the areas of low contrast which is destroyed by the noise. The second step is related to the presence of the shrinkage wavelets which has reduced the still existing noise. Coupled with the BDBM model, we were able to improve both CNR and tissue texture accuracy.

In contrast, the OBDBM technique does not participate by any filter, which means that the estimate comes with a lower CNR because of the large distribution of the noise that affects the CNR criterion.

Also, the monogenic technique has provided a low CNR in front of our proposed method; this is because of the implemented DoG filter having not succeeded in reducing the noise present in the high frequency component [21].

According to Table 3, the OBDBM method achieves the lowest SSIM measurement value; therefore this technique provides a less similar structure image. This is very logical since this technique does not contain a filter to correct the details deterioration caused by the noise. The monogenic technique has somewhat higher SSIM values than the OBDBM method; however it does not work well when it is compared to the proposed technique. This is explained by the DoG filtering of the monogenic technique, which did not filter the noise properly. The higher SSIM values are obtained for the proposed method, and the results are encouraging. We can easily notice that the coupling between the FBP and the shrinkage wavelets combined with BDBM model has improved the image quality and has provided a good results in SSIM values than other techniques.

The proposed approach has a more similar structure of the tissue displacement estimation images; it is the most effective and the most efficient.

The implementation of the adopted strategy in clinical evaluation of mammary pathologies, allows improving the results of the OBDBM model used in estimating the breast tissue deformation, the strategy of image reconstruction coupled to shrinkage wavelets and combined with BDBM model makes it possible to optimize the clinical diagnosis.

Considering all this, the proposed method can be a very powerful tool for the improvement of breast tissue displacements images, against details deterioration and loss of the contrast caused by speckle noise. The implementation of the proposed approach will help to ensure a reliable and accurate diagnosis in static ultrasound elastography.

\section{Conclusion}

A new method of breast tissue displacements estimation analysis has been proposed in this paper. The proposed strategy optimized the OBDBM technique used to estimate the displacements estimation in ultrasound breast elastography. Our adopted approach is based on the coupling of FBP and shrinkage wavelets combined with the BDBM model in order to improve the diagnosis of breast pathology.

Implementation of our proposed method is discussed, using 20 B-mode images corresponding to 20 patients having breast tumors and it has been found that the proposed technique reduces perfectly the noise and enhances image detail, giving better SD, higher CNR and more appropriate SSIM when compared to the OBDBM and monogenic techniques.

Consequently, the proposed method gives good results and its implantation in the elastography imaging systems will definitely improve the clinical evaluation and the diagnosis of pathological breast tissues.

\section{References}

1. Xiao Y, Yu Y, Niu L, Qian M, Deng Z, et al. (2016) Quantitative evaluation of peripheral tissue elasticity for ultrasound-detected breast lesions. Clin Radiol 71: 896-904.

2. Park CS, Kim SH, Jung NY, Choi JJ, Kang BJ, et al. (2015) Interobserver variability of ultrasound elastography and the ultrasound BI-RADS lexicon of breast lesions. J Breast Cancer Res. 22: 153-160.

3. Wang ZG, Liu Y, Wang G, Sun LZ (2009) Elastography method for reconstruction of non-linear breast tissue properties. Int J Biomed Imaging 2009: 1-9.

4. Ricci P, Maggini E, Mancuso E, Lodise P, Cantisani V, et al. (2014) Clinica application of breast elastography: State of the art. Eur J Radiol. 83: 429-437.

5. Mittal A, Soundararajan R, Bovik AC (2013) Making a completely blind image quality analyzer. IEEE Signal Process 20: 209-212.

6. Lowerison MR, Hague MN, Chambers AF, Lacefield JC (2016) Improved Linear Contrast-Enhanced Ultrasound Imaging via Analysis of First-Order Speckle Statistics. IEEE Trans Ultrason Ferroelectr Freq Control 63: 1409-1421.

7. Gu P, Lee WM, Roubidoux MA, Yuan J, Wang X, et al. (2016) Automated 3D ultrasound image segmentation to aid breast cancer image interpretation. Ultrasonics 65: 51-58.

8. Maltaverne T, Delachartre P, Basarab A (2010) Motion estimation using the monogenic signal applied to ultrasound elastography. Conf Proc IEEE Eng Med Biol Soc 10: 33-36.

9. Coupé P, Hellier P, Kervrann C, Barillot C, et al. (2009)Nonlocal means-based speckle filtering for ultrasound Images. IEEE Trans Image Process 18: 2221-2229.

10. Thomas A, Kümmel S, Fritzsche F, Warm M, Ebert B, et al. (2006) Realtime sonoelastography performed in addition to B-mode ultrasound and mammography: Improved differentiation of breast lesions. Acad Radiol 13 1496-1504.

11. Chetih N, Messali $Z$ (2015) Tomographic image reconstruction using filtered back projection (FBP) and algebraic reconstruction technique (ART). 3rd International Conference on Control, Engineering and Information Technology.

12. Kuo Y, Lin YY, Lee RC, Lin CJ, Chiou YY, et al. (2016) Comparison of image quality from filtered back projection, statistical iterative reconstruction, and model-based iterative reconstruction algorithms in abdominal computed tomography. Medicine (Baltimore) 95: 31.

13. Pérez-Liva M, Herraiz JL, Medina-Valdés L (2015) Regularization of image reconstruction in ultrasound computed tomography. 2015 IEEE Nuclear Science Symposium and Medical Imaging Conference (NSS/MIC), pp: 1-3.

14. Slimi T, Moussa IM, Kraiem T, Mahjoubi H (2017) Improvement of displacement estimation of breast tissue in ultrasound elastography using the monogenic signal. Biomed Eng Online 16: 19

15. Basarab A, Aoudi W, Liebgott H, Vray D, Delachartre P (2007) Parametric deformable block matching for ultrasound imaging. Proc IEEE Int Conf Image Process, pp: 429-432.

16. Seferidis V, Ghanbari M (1994) Generalised block-matching motion estimation using quad tree structured spatial decomposition. IEE Proc Vision Image Signal Process 141: 446-452.

17. Wei W, Hou ZX, Guo YCG (2005) A displacement search algorithm for deformable block matching motion estimation. Proc IEEE Int Symp Commun Inf Technol 457-460.

18. Zhu Y, Hall TJ (2002) A modified block matching method for real-time freehand strain imaging. Ultrasonic Imaging 24: 161-176.

19. Alessandrini M, Bernard O, Basarab A, Liebgott $H$ (2013) Multiscale optical computation from the monogenic signal. AEMB 34: 33-37.

20. Unser M, Sage D, Van De Ville D (2009) Multiresolution monogenic signa analysis using the Riesz-Laplace wavelet transform. IEEE Trans Image Process 18: 2402-2418.

21. Prabusankarlal KM, Thirumoorthy P, Manavalan R (2015) Assessment of combined textural and morphological features for diagnosis of breast masses in ultrasound. $\mathrm{HCIS} \mathrm{5:} \mathrm{1-17.}$ 